\title{
Successful Application of Ultrasonic Therapy
}

\author{
(Die praktische Bewährung der Ultraschalltherapie)
}

\author{
$B y$ Dr med. K. STUHFLAUTH
}

$\mathbf{U}^{1}$ trasonic therapy developed from an idea of the physicist Pohlman in 1939 to utilize mechanical vibrations of high frequency for the treatment of rheumatic diseases.

After the war an apparatus for applying this type of therapy was developed, and its use spread within a few years from Germany, via Switzerland, Italy, France and Spain to almost all the countries of the world. During this time contradictory opinions were expressed by the German medical public concerning ultrasonic therapy. After the first enthusiasm subsided, a time followed in which this method was looked upon sceptically and often even met with a negative attitude which in many cases was unfounded. The appraisal of ultrasonic therapy by the medical world followed thus exactly the same pattern as that of X-ray therapy. After X-ray therapy had been enthusiastically received it was almost declared illegal during the 1920's on account of the dangers involved.

As a result of the negative attitude towards ultrasonic therapy the German Health Insurance Companies refused to refund payments for it. This decision was amended only in April 1954 when upon the suggestion of Professor von Braunbehrens the previous decision was reversed because a number of European countries had introduced ultrasonic therapy and acknowledged it as acceptable for Insurance Companies.

The exaggerated fears of the so-called ultrasonictherapy - which by the way, until today did not cause one single death-was the result of observations of undesirable effects in animal experiments, in treatment of patients with an overdosage and in cases where such treatment was contraindicated.

Results from research with ultrasonic vibrations using isolated tissues or living animals cannot as such be applied to the human. They will only provide an interesting insight into the action of this therapy. The pediatrician Adam for instance reports 1500 single applications of ultrasonics in children without any case of the bone damage being observed even though Buchtala, Barth and Bullow clearly describe bone damage and interference of growth when treating young dogs. In all animals dosages were used which by far surpassed the therapeutic safe margin, and which usually, were applied with the stationary method of application. The following tests show how important it is to consider the type of application for accurate evaluation of the results: Maibaner reports application of ultrasonic therapy on guinea-pigs using the massaging method with dosages used in treatment of human beings. In not even one single case did he observe skin damage. Baumann and Presch using the same dosage, however, with the stationary method, observed edema, blisters and finally even tissue destruction. Klinkmüller observed the effect of ultrasonic therapy on a leg which was scheduled for amputation. Definite tissue damage was only found in those spots which were exposed for 20 minutes to stationary sonic vibrations.

The empirically determined dosages now used are far below those used from 1947 to 1949 . Hintzelmann for instance lists in his dosage table radiating intensities from 0,2 to a maximum of 3 watt $-\mathrm{cm}^{2}$. An increase of this dosage for the purpose of obtaining a better therapeutical effect often results in untoward-effects, such as pains and spaism.
I mentioned already in 1950 that irradiation with the previously used high dosages caused an imbalance of the autonomic nervous system in very sensitive persons and that circulatory disturbances such as angina pectoris attacks, dizzy spells caused by drop of blood pressure etc., may occur.

The findings were confirmed repeatedly by Pezold, Bunse and co-workers, as well as Leicht and co-workers. It is furthermore of interest that such symptoms, as reported by other authors, can also be observed after X-ray therapy when larger vegetative nerve cords or ganglia are directly affected by the radiating energy (Arkussy, Günsel and Stefanow). With the low ultrasound dosages now used, one does not have to refrain from treating older or circulatory-labile persons with ultrasonic therapy. We can report from experiences gathered at our clinic that with a maximum radiation intensity of 2 Watt $-\mathrm{cm}^{2}$ and an average radiation intensity of $0,3-0,5$ Watt $-\mathrm{cm}^{2}$ such symptoms no longer occur. A direct sonic treatment however, of the precordial area and the cervical ganglia should be avoided when treating persons with a history of heart discease.

It is important to use this newly developed therapy discriminately and to apply only in cases where it is indicated. Many errors have been made in this respect, partly due to exaggerated claims, partly due to unjustified conclusions from animal experimentation. Another error was made by theoretically comparing this form of therapy with other methods of physical therapy.

A good example of this is the question of sonic treatment of tumors. From biological experiments we can state, that similar results were obtained with X-ray and ultrasound, while the effectiveness differed greatly. According to information obtained from Wallace, Bushnell and Newcomer the effectiveness of ultrasonic waves may be rightfully compared with that of electromagnetic or corpuscular ionizing rays. The same damage curve was plotted with ultrasonic and X-ray treatment of drosophila melanogaster. Newcomer and Wallace observed mutation processes when treating corn, while Asche clearly discovered "Colchicin effects" on plant cells when in the process of cell division, and Woeber found with the WalkerCarcinoma caryorhexis and carolysis which destroy the tumor tissue. However, the fact that in spite of all this knowledge tumors have never been treated successfully in human beings is due to the too low ultrasound intensities used which also may lead to an opposite-effect. We found it to be true that too low intensities - not only of ultrasound but also of ionizing rays - can rather lead to an increased growth in patients with tumors in areas which were not readily accessible to a sound beam of sufficient strong intensity. We therefore suggest to refrain from sound treatment of tumors, since rapid growth of metastases was observed after sound treatment. Unless such treatment could be combined with simultaneous X-ray therapy, which-according to tests by Woeber-results in sensitizing of the cells toward X-rays.

Unpertubed by the heated discussion pro and contra the effect of ultrasonic therapy, we find that many clinics and almost all institutes of physical therapy have included ultrasonic therapy into their armamentarium of medical treatment, as well as short wave therapy, X-ray, ultraviolet 
light treatments, etc. Clinical experience now permits light exact indications of ultrasonic therapy for successful treatment of various diseases. Statistical data were comniled during the last year by the "Scientific Association for Ultrasonic Research. "285 practitioners and specialists and also 27 clinics participated in this survey, and of the 120,000 individually treated cases a total of 103,000 were evaluated $(W u l f)$. The following indications are listed according to the effectiveness of ultrasonic therapy:

Statistics of recovery of the group including more than 100 cases treated, (Total number of cases treated 103,360) arranged according to tresult obtained in $\%$.

\begin{tabular}{lllll} 
Recovery groups & I & II & III & IV \\
\hline Lumbago & 74 & 25 & 1 & - \\
Myalgia & 72 & 26 & 2 & - \\
Contusions & 62.5 & 35.5 & 1 & 1 \\
Distortions & 58.5 & 34.5 & 7 & - \\
Inflammatory skin lesions & 57.5 & 41 & 1.5 & - \\
Sciatica & 55.5 & 42 & 2.5 & - \\
Periarthritis & 41.5 & 48 & 10.5 & - \\
Herpes zoster & 39 & 44 & 14.5 & 2.5 \\
Tenosynovitis & 36.5 & 58 & 5.5 & - \\
Chilblains & 34 & 61 & 5 & - \\
Stomach Ulcers & 32.5 & 44.5 & 23 & - \\
Arthritis & 32.5 & 56 & 9.5 & 2 \\
Raynaud's Disease & 30.5 & 31.5 & 38 & - \\
Epicondylitis & 30 & 36.5 & 33.5 & - \\
Sudeck's Atrophy & 28.5 & 53.5 & 18 & - \\
Plexus Neuralgia & 28 & 54.5 & 16.5 & 1 \\
Buerger's Disease & 23.5 & 36 & 36 & 4.5 \\
Arthrosis def, & 27 & 61 & 12 & - \\
Brachialgia noct, & 21 & 50.5 & 28.5 & - \\
Stump Neuroma & 21 & 53.5 & 25.5 & - \\
Spondylarthrosis & 20 & 58.5 & 21.5 & - \\
Ulcus cruris & 18.5 & 52 & 18.5 & 1 \\
Warts & 18 & 22 & 60 & - \\
Prostatitis & 16 & 53 & 31 & - \\
Sclerodermia & 14.5 & 31 & 54.5 & - \\
Marie-Strumpell Disease & 14.5 & 62.5 & 23 & - \\
Cholecystopathy & 13 & 70.5 & 16.5 & - \\
Asthma & 11.5 & 51.5 & 35 & 2 \\
$\quad$ I = very good success & & & & \\
\multicolumn{1}{|l}{ II = good success. } & & & & \\
$\quad$ III = no or rarely success. & & & & \\
$\quad$ IV deterioration. & & & & \\
\hline
\end{tabular}

\section{Main indications for Ultrasonnic Therapy.}

Arthrosis and spondylarthrosis with rheumatic and neuralgic manifestations such as sciatica, lumbago, muscular rheumatism, periarthrit is humeroscapularis, plexus neuralgia, myalgia etc., are the main indications for ultrasonic therapy. of 103,000 cases treated and evaluated, $55 \%$ showed very good results and $38 \%$ good results. The physicians reported the application of ultrasound the method of choice in these cases. On the other hand we find that acute inflammatory arthritis and advanced cases of Bechterew disease react less favourably to ultrasound treatment. Excellent results are for instance reported by Reichel in treating contusions and sprains, dislocations etc. (60\% very good results; $30 \%$ good results). Frey, of Bern, Switzerland, successfully treated the Swiss olympic team during the olympic games in Helsinki with ultrasound. Edema and other swellings resulting from sprains and fractures (Zauner) disappear much faster with ultrasonic treatment. Inflammatory skin conditions, such as furuncles, or glandular abscesses can be treated successfully. The resorption of inflammatory indurations, for instance after tooth extractions, is greatly accelerated. According to our own experiences and to reports given by others, radicular ultrasound treatment is very effective, especially in cases of severe pain in herpes zoster, even when other therapeutic methods have failed. Ultrasound here may be considered the method of choice. Similar good results were reported by clinics and practitioners in causalgias (Chateau), coccygodynia (Forestier), postoperative neuritis and neuralgia of the jaw (Herrmann). Behrend of New York emphasizes diminution of rigor, spasm and pain in hemiplegia, Parkinson's disease and multiple sclerosis after radicular sound treatment, Less good results in spite of initially enthusiastic reports can be obtained in Sudeck's atrophy, ulcus cruris, bronchial asthma and sclerodermia. These good results reported from Germany, Switzerland and Italy are now confirmed by large ststistics originating in U.S.A., where Aldes alone treated more than 170 cases of subdeltoid bursitis and Behrend reported a total of more than 10,000 treatments. Most of the indications for ultrasonic therapy mentioned above are also valid for the use of other orthodox modalities of physical therapy. Many of the diseases quoted have already previously been successfully treated with analgesic dosages of X-ray therapy, for instance arthralgia and arthritis, inflammatory conditions of the skin and inflammation of the lymph glands. X-ray radiation of the sympathetic chain and of the ganglia for treatment of angio-spastic symptoms has been used for a long time and is considered to replace the drastic sympathectomy according to Leriche. This invites comparison with paravertabral sound treatment which according to Stuhlfauth and associates has almost the pharmacological effect of a mild novocain-block.

Painful rheumatic afflictions of the muscles and neuralgia are successfully treated by practitioners with short wave therapy. It is now up to the physician to choose from the many therapeutical methods offered, to which ultrasonic therapy has recently been added. His final decision will depend on the prevailing circumstances in each individual case.

Comparative tests were made by Dalicho in treating joint diseases. Dalicho compared ultrasound therapy with $X$-ray therapy. in treating arthralgia. He found that the results obtained with ultrasonic therapy are almost identical with those of X-ray therapy. This compares with a report by Wallace and associates on animal experimentation with drosophila melanogaster. On the other hand the results obtained are much more favourable as when only thermal therapy was used. When treating larger joints which are not so readily accessible to ultrasonics results are less favourable. The percentage of hip joints treated successfully with X-ray therapy is $73 \%$ compared to only $44 \%$ when treated with ultrasound (Pannewitz). One advantage of ultrasonic therapy over X-ray therapy is its low cost which permits its use by any physician. It further permits well localized treatment of smallest areas and it is specially recommended for treatment of the readily reacting autonomic nervous system (Stuhlfauth, Zach).

The advantage of X-ray therapy consists in its greater intensity and degree of penetration. The choice between these two therapeutic modalities therefore depends on whether superficial or deeper located areas of the body or deep-seated centres, joints or inflammatory foci are to be treated.

\section{The action of Ultrasound.}

The action of ultrasound is not yet fully understood, which also holds true for many other modalities of physical therapy. It is still under discussion whether or not the primary effect of ultrasound is merely a thermal one, and to which extent other mechanical or chemical effects enter into the picture. Lehmann from the very beginning offered the theory that ultrasound has merely a well localized thermal effect. He is supported in his theory by many scientific experiments. Meanwhile Schmitz and associates, Halbach, Stuhlfauth, Goelkel and Meyer claim mechanical physical effects, especially on the muscle and its membrane- 
According to theories established so far we find that the decisive factor for release of the primary processes which produce the radiation lies in the local accumulation of energy which releases chemical reactions. Compression and decompression of tissue leads to powerful energyconcentration in localised areas which in turn release various reactions. Numerous physical processes take place such as division, synthesis, oxidation, reduction and polymerization.

The X-ray effect is explained by a mechanism which can be experimentally confirmed (Wels, Dale and others, Barron and his colleagues). When irradiating water, both $\mathrm{OH}$-radicals and $\mathrm{H}$-atoms are generated which combine to $\mathrm{H}_{2} \mathrm{O}_{2}$ (Langendorff). Such reactive groups lead to oxidation of the SH-group in the cell proteins thereby blocking enzymes. In serious cases, a denaturation of albumen may set in. A similar mechanism could be responsible for the primary effect of ultrasound on the cell structure.

The therapeutic effect, however, principally results from secondary effect which is transmitted via the autonomic nervous system. Application for an appropriate dosage results in relaxation of the smooth and striated muscles, in an increased blood circulation and a locally effective relief of pain. Other long known factors are the heating effect of the tissue (Pohlman), the increase of the blood circulation of the skin (Stuhlfauth) and the electromyographically recorded decrease of the muscle tone (Tschannen).

Ultrasound combined with other therapeutic measures.

Since ultrasound must not be considered a panacea, physiatrists combined ultrasound with other therapeutic methods. Kowarschick combines ultrasound with massage, Hintzeman with massage of the connective tissue, Ladeburg with underwater massage, Forestior with thermal baths, Gross with steam baths, Koeppen with short wave treatment, thereby obtaining much better results. Sound treatment after local application of ointments, such as Finalgon (Ohlig) and Venostasin (Maennel) contributes to the subjective relief of pain.

In very painful conditions of the joint and afflictions of the spine (such as lumbago and sciatica) we employ a combination of several modalities, especially designed for easy use by the practitioner. Half an hour prior to the first ultrasound treatment an Irgapyrin injection is given which assists in procuring central analgesia and relaxation. Simultaneously, a series of injections with the GT 50 (one vial $1-2$ times per week) is started, the high vitamine $D_{2}$ content in combination with other effective substances (vagomimetica, hormones) evidently helps to regenerate the osteochondrotic and deformed joint to a certain extent.

Relaxation of the joints, simultaneously results in a trophic influence on the regeneration of the supporting connective tissue. Success of ultrasonic therapy depends on correct indication and dosage, combined with patience. I have received enthusiastic reports from medical husbandwife teams where the wife assisted with skillful hands and great patience in the treatment of patients. Nervous and rushed physicians who hardly have the time to supervise their thchnicians will not obtain good results, and will then blame ultrasonics rather than its faulty application and technic for their failures.

\section{Conclusion}

Developments in ultrasonic therapy during the past 7 years are reviewed in order to promote better appreciation of this type of therapy which may be classified under vibratory-massaging procedures.

A period of great enthusiasm was soon followed in Germany by an actually too sceptical attitude which later was reversed by including ultrasonic therapy into the list of treatments accepted by Health Insurance Companies. In contrast to X-ray therapy which in the 1920's underwent a similar course of developments, ultrasonic therapy is not known yet to have caused any damaging effect. Reactive effects, such as pain, exacerbations, spasm of blood vessels disappeared after proper adjustment of the dosage. Whereas during the past few years practitioners in Germany refrained from using ultrasonic therapy, it has now become widely accepted all over Europe, in South America and finally in North America where it is considered an effective method of treatment for rheumatic and traumatic disorders and in rehabilitation procedures. Extensive European and International Congresses and reports from America testify to this effect. German Institutes of physical therapy, clinics and hospitals as well as practitioners have now familiarized themselves throughly with the manual method of treatment and have reported very favourable results. Based on German statistics of more than 120,000 treated cases and based on statistics reported by the U.S.A., a list of indications was compiled. In conclusion, the author demonstrates how other modalities of physical and drug therapy in combination with ultrasonic therapy speed up recovery, especially in cases of chronic rheumatic diseases and in rehabilitation procedures. Such a combined method not only accelerates the process of healing, but at the same time prevents any possible relapses.

\section{Deliverance}

from pain . . . the humanitarian function of of the practitioner.... now accomplished with greater speed, more economy and with a higher degree of safety than ever before.

Full details and clinical notes on the use of

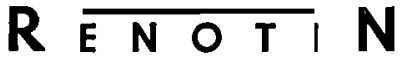

in the treatment of Migraine, Neuritis, Lumbago, Sprains and Myalgía may be obtained from

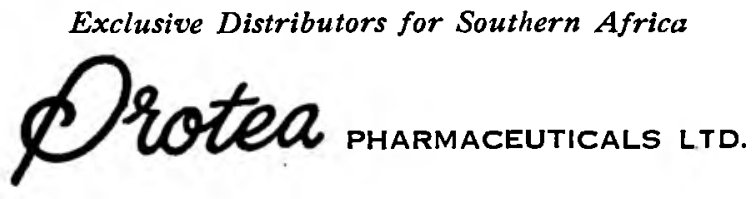

P.O. BOX 7793 JOHANNESBURG 